\title{
A NOTE ON THE DIMENSION OF THE LARGEST SIMPLE HECKE SUBMODULE
}

\author{
SANDRO BETTIN, CORENTIN PERRET-GENTIL, AND MAKSYM RADZIWIE€
}

\begin{abstract}
For $k \geq 2$ even, let $d_{k, N}$ denote the dimension of the largest simple Hecke submodule of $S_{k}\left(\Gamma_{0}(N) ; \mathbb{Q}\right)^{\text {new }}$. We show, using a simple analytic method, that $d_{k, N} \gg_{k} \log \log N / \log (2 p)$ with $p$ the smallest prime co-prime to $N$. Previously, bounds of this quality were only known for $N$ in certain subsets of the primes. We also establish similar (and sometimes stronger) results concerning $S_{k}\left(\Gamma_{0}(N), \chi\right)$, with $k \geq 2$ an integer and $\chi$ an arbitrary nebentypus.
\end{abstract}

\section{InTRODUCTION}

For an integral weight $k \geq 2$ and a level $N \geq 1$, the anemic Hecke $\mathbb{Q}$-algebra

$$
\mathbb{T}:=\mathbb{Q}\left[T_{n}:(n, N)=1\right],
$$

generated by the Hecke operators $T_{n}$, acts on the space of cusp forms $S_{k}\left(\Gamma_{0}(N)\right)$.

Simple Hecke submodules of $S_{k}\left(\Gamma_{0}(N)\right)$ of dimension $d$ correspond to $\operatorname{Gal}(\overline{\mathbb{Q}} / \mathbb{Q})$ orbits of size $d$ of (arithmetically) normalized eigenforms $f \in S_{k}\left(\Gamma_{0}(N)\right)$. When $k=2$, the work of Shimura also gives a correspondence with simple factors of dimension $d$ of the Jacobian $J_{0}(N)$ of the modular curve $X_{0}(N)$. Thus it is interesting to ask about the dimension $d_{k, N}$ of the largest simple Hecke submodule of $S_{k}\left(\Gamma_{0}(N)\right)$, or equivalently the maximal degree of Hecke fields of normalized eigenforms.

Maeda [HM97] postulated that $S_{k}\left(\Gamma_{0}(1)\right)$ is a simple Hecke module for all even $k \geq 12$. This deep conjecture implies among other things that $L\left(\frac{1}{2}, f\right) \neq 0$ for all $f \in S_{k}\left(\Gamma_{0}(1)\right)$, see [CF99]. When $N>1$, there is an obstruction to simplicity due to the Atkin-Lehner involutions, but numerical evidence suggests that this is the only asymptotic barrier when $N$ is square-free. This led Tsaknias [Tsa14] to suggest the following generalization of Maeda's conjecture (see also [DT16] for non-square-free levels):

Conjecture 1. For $k \geq 2$ even and large enough and $N$ square-free, the number of Galois orbits of newforms in $S_{k}\left(\Gamma_{0}(N)\right)$ is $2^{\omega(N)}$. In particular, for any fixed $\varepsilon>0$ we have

$$
d_{k, N} \gg_{k, \varepsilon} N^{1-\varepsilon} .
$$

That is, there exists a constant $c(k, \varepsilon)>0$ depending at most on $k$ and $\varepsilon$ such that $d_{k, N}>c(k, \varepsilon) N^{1-\varepsilon}$ for all square-free $N \geq 1$. 
There is a massive gap between Conjecture 1 and the unconditional results. Through an equidistribution theorem for Hecke eigenvalues, Serre [Ser97] was the first to establish that $d_{k, N} \rightarrow \infty$ as $k+N \rightarrow \infty$. Subsequently, by making Serre's equidistribution theorem effective, Royer [Roy00] and Murty-Sinha [MS09] showed that $d_{k, N} \gg_{k, p} \sqrt{\log \log N}$ for any $p \nmid N$. In the particular case where $N$ lies in a restricted set of primes, this bound has been improved by several authors. Extending a method of Mazur to all even weights, Billerey and Menares [BM16, Theorem 2] obtained that $d_{k, N} \gg_{k} \log N$ when $N \geq(k+1)^{4}$ is in a explicit set primes of lower natural density $\geq 3 / 4$. When the lower bound is fixed in advance and one looks for a level with a given number of prime divisors attaining it, see also [DJUR15]. When $N \equiv 7(\bmod 8)$ is prime, Lipnowski-Schaeffer [LS18, Corollary 1.7] also showed that $d_{2, N} \gg \log \log N$, which can be significantly improved for $N$ in certain subsets of the primes under certain well-known conjectures and heuristics.

In this paper we show that bounds of Lipnowski-Schaeffer quality can be obtained for all levels and integer weights. Our method is however, analytic and we believe simpler than the one in [LS18].

Theorem 1. Let $k \geq 2$ even and $N \geq 1$ be integers. Then the dimension of the largest simple Hecke submodule of $S_{k}\left(\Gamma_{0}(N)\right)^{\text {new }}$ is

$$
d_{k, N} \gg_{k} \frac{\log \log N}{\log \left(2 p_{N}\right)}
$$

as $N \rightarrow \infty$, where $p_{N}$ denotes the smallest prime co-prime to $N$.

Since the vast majority of integers $N$ have a small co-prime factor, this bound is essentially asserting that $d_{k, N} \gg_{k} \log \log N$. Theorem 1 appears to be the first bound of " $\log \log N$ strength" for any even weight $k \geq 4$, and in the case $k=2$, without restriction on the level.

We state below a more general and precise form of Theorem 1 that holds in the presence of a nebentypus.

Theorem 2. Let $k \geq 2$ and $N \geq 1$ be integers. Let $p \nmid N$ and let $\chi:(\mathbb{Z} / N)^{\times} \rightarrow \mathbb{C}^{\times}$ be a homomorphism such that $\chi(-1)=(-1)^{k}$. Then the maximum size of the $\operatorname{Gal}(\overline{\mathbb{Q}} / \mathbb{Q})$-orbits of newforms $f \in S_{k}\left(\Gamma_{0}(N), \chi\right)$ is

$$
\geq \frac{2}{(k-1) \log (4 p)} \cdot \log \left(\frac{\log N}{2 \pi \log p}\right)
$$

for all sufficiently large $N$ (in terms of $k$ ).

By definition, the same lower bound holds for the maximum degree of the Hecke fields $K_{f}$ of newforms $f$ (see Section 2). Note that $K_{f}$ always contains the cyclotomic 
field $\mathbb{Q}\left(\zeta_{\text {ord }}(\chi)\right)$ generated by the values of $\chi$ (a consequence of the Hecke relations at $p^{2}$, see Lemma 2 below), so the trivial lower bound in both cases is $\varphi(\operatorname{ord}(\chi))$.

Remark 1. The result of Billerey-Menares mentioned above actually shows that when $\ell \geq(k+1)^{4}$ belongs to an explicit set $\mathcal{L}$ of primes with lower density $\geq$ $3 / 4$, there exists a normalized eigenform $f \in S_{k}\left(\Gamma_{0}(\ell)\right)^{\text {new }}$ with $\operatorname{deg}\left(K_{f}\right) \gg_{k} \log \ell$. Hence, for $\varepsilon>0$, if an integer $N$ has a prime factor $\ell>N^{\varepsilon}$ that lies in $\mathcal{L}$, then $\operatorname{deg}\left(K_{f}\right) \gg_{k, \varepsilon} \log N$ for some $f \in S_{k}\left(\Gamma_{0}(\ell)\right) \hookrightarrow S_{k}\left(\Gamma_{0}(N)\right)$. Hence, Theorem 2 with "newform" replaced by the weaker conclusion "normalized eigenform" would follow from [BM16, Theorem 2] for almost all integers $N$.

In certain special situations it can be shown that the degree of the number field $K_{f}$ is large for all newforms $f \in S_{k}\left(\Gamma_{0}(N), \chi\right)$. For instance, when $p^{r} \mid N$ Brumer [Bru95, p.3, Theorem 5.5, Remark 5.7] showed that $K_{f}$ contains the maximal real subfield of the $p^{s}$-th roots of unity, where $s=\left\lceil\frac{r}{2}-1-\frac{1}{p-1}\right\rceil$ (see also [Mat10, CE04]).

We exhibit a similar phenomenon which sometimes allows to significantly improve on Theorem 2 and the trivial bound $\operatorname{deg} K_{f} \geq \varphi(\operatorname{ord} \chi)$, when $k$ is odd, depending on the nebentypus $\chi$ and the factorization of $N$.

Theorem 3. Let $k \geq 3$ be an odd integer, $N \geq 1$ be square-free, $\chi:(\mathbb{Z} / N)^{\times} \rightarrow \mathbb{C}^{\times}$ be a homomorphism such that $\chi(-1)=(-1)^{k}$, and decompose

$$
N_{2}=\prod_{\substack{p \mid N \\ \chi_{p}=1}} p, \quad \chi=\prod_{p \mid N} \chi_{p}, \quad \text { with } \quad \chi_{p}:(\mathbb{Z} / p)^{\times} \rightarrow \mathbb{C}^{\times} .
$$

Then, for any newform $f \in S_{k}\left(\Gamma_{0}(N), \chi\right)$,

$$
\operatorname{deg} K_{f} \geq \varphi(\operatorname{ord}(\chi)) \cdot 2^{\omega\left(N_{2}\right)-\omega\left(\left(N_{2}, 2 \operatorname{ord}(\chi)\right)\right)-1},
$$

In particular, if $\left(N_{2}, 2 \operatorname{ord}(\chi)\right)=1$, then

$$
\operatorname{deg} K_{f} \geq \varphi(\operatorname{ord}(\chi)) \cdot 2^{\omega\left(N_{2}\right)-1} .
$$

For example, given $\varepsilon>0$ and $k \geq 3$ odd, for a "typical" square-free integer $N$ and $\chi$ a random quadratic character mod $N$ (resp. the trivial character), we get

$$
\operatorname{deg} K_{f} \gg_{\varepsilon}(\log N)^{\frac{\log 2}{2}-\varepsilon} \quad\left(\text { resp. } \gg_{\varepsilon}(\log N)^{\log 2-\varepsilon}\right)
$$

for all newforms $f \in S_{k}\left(\Gamma_{0}(N), \chi\right)$. In fact it is possible to extend Theorem 3 to the case of non-square-free $N$, but we maintain this restriction to keep the exposition simple. 
A short outline of the proofs. We will now say a few words about the proof of these theorems and the limitations of our method of proof.

The proof of Theorem 1 and Theorem 2 proceeds by observing that if we can find a newform $f$ for which the eigenvalue $a_{f}\left(p_{N}\right)$ is abnormally small in absolute value but non-zero, then the degree of the corresponding Hecke field $K_{f}$ needs to be large (see Proposition 1). We then use the equidistribution of Hecke eigenvalues (in the form of Murty-Sinha) to prove the existence of such an $f$. This contrasts with the previous analytic approaches in which one probed (using the equidistribution of Hecke eigenvalues) the neighborhood of every algebraic integer up to a certain height.

The proof of Theorem 3 proceeds by first noticing that by strong multiplicity one, the number field $\mathbb{Q}\left(a_{f}(n): n \geq 1\right)$ coincides with $K_{f}=\mathbb{Q}\left(a_{f}(n):(n, N)=1\right)$. Subsequently we focus exclusively on the ramified primes $p \mid N$. For $k$ odd, the coefficient of $f$ at $p \mid N_{2}$ is equal to $\sqrt{p}$ multiplied by a factor lying in a small extension of $K_{f}$ (the eigenvalue of an Atkin-Lehner operator). Considering all these divisors yields the factor $2^{\omega\left(N_{2}\right)}$.

Limitations of the method. The best result that the method of proof of Theorem 1 and Theorem 2 can theoretically deliver is for each $k$ even and $N \geq 1$ the existence of an $f \in S_{k}\left(\Gamma_{0}(N)\right)$ such that $\operatorname{deg} K_{f} \gg_{k} \log N$. To see this consider for simplicity $k$ fixed and $N$ odd. Then we expect that the coefficients $a_{f}(2)$ with $f$ varying in $S_{k}\left(\Gamma_{0}(N)\right)$ behave as a collection of roughly $\asymp_{k} N^{1+o(1)}$ random numbers distributed according to the Sato-Tate law. Therefore by linearity of expectation for any given $\varepsilon>0$ we expect that there exists a form $f \in S_{k}\left(\Gamma_{0}(N)\right)$ with $0<\left|a_{f}(2)\right| \ll_{k}$ $N^{-1+\varepsilon}$ and moreover that this is best possible up to the factor $N^{\varepsilon}$. Plugging this into Proposition 1 would result in a lower bound $\operatorname{deg} K_{f} \gg_{k} \log N$ for some $f \in$ $S_{k}\left(\Gamma_{0}(N)\right)$. Note that the existence of a $\delta>0$ such that for all $k$ fixed and $N$ odd there exists an $f \in S_{k}\left(\Gamma_{0}(N)\right)$ with $0<\left|a_{f}(2)\right| \ll_{k} N^{-\delta}$ would be also enough to obtain the lower bound $\operatorname{deg} K_{f} \gg_{k} \log N$ for some $f \in S_{k}\left(\Gamma_{0}(N)\right)$.

Acknowledgments. The work of the first author is partially by PRIN 2015 "Number Theory and Arithmetic Geometry". The third author would like to acknowledge the support of a Sloan fellowship. We would like to thank Nicolas Billerey, Armand Brumer, and Ricardo Menares for comments on the manuscript. We would like to thank the referees for a careful reading of the paper and useful suggestions.

\section{Proof of Theorem 1 And Theorem 2}

Throughout let $k \geq 2$ and $N \geq 1$ be integers, and $\chi:(\mathbb{Z} / N)^{\times} \rightarrow \mathbb{C}^{\times}$a homomorphism such that $\chi(-1)=(-1)^{k}$. Let $f \in S_{k}\left(\Gamma_{0}(N), \chi\right)$ be a normalized eigenform 
with Fourier expansion

$$
f(z):=\sum_{n \geq 1} a_{f}(n) e(n z), \quad a_{f}(1)=1, \quad e(z):=e^{2 \pi i z} .
$$

Given a prime $p \nmid N$, we also define (for reasons that will become clear when proving Lemma 1)

$$
a_{f}^{\prime}(p)=\frac{a_{f}(p)}{2 p^{\frac{k-1}{2}} \sqrt{\chi(p)}} \in \mathbb{R},
$$

for a fixed choice of square root.

Since simple Hecke submodules of $S_{k}\left(\Gamma_{0}(N)\right)$ of dimension $d$ correspond to $\operatorname{Gal}(\overline{\mathbb{Q}} / \mathbb{Q})$ orbits of size $d$ of (arithmetically) normalized eigenforms $f \in S_{k}\left(\Gamma_{0}(N)\right.$ ) (see [DI95]), it suffices to obtain lower bounds for

$$
\max _{f \in S_{k}\left(\Gamma_{0}(N), \chi\right)} \operatorname{deg} K_{f}, \quad K_{f}=\mathbb{Q}\left(a_{f}(n):(n, N)=1\right),
$$

where $f$ runs over newforms, to prove Theorems 1 and 2 .

The first input to our argument is a simple lemma from diophantine approximation, that allows to pass from small values of $\left|a_{f}(p)\right|$ to lower bounds for the degree of the Hecke field.

Proposition 1. If $p \nmid N$ and $a_{f}(p) \neq 0$, then

$$
\operatorname{deg} \mathbb{Q}\left(a_{f}(p)\right) \geq \frac{2}{k-1} \cdot \frac{\log \frac{1}{\left|a_{f}^{\prime}(p)\right|}}{\log (4 p)} .
$$

Proof. Since $a_{f}(p)$ is an algebraic integer [D195, Corollary 12.4.5], its norm is a nonzero integer. Thus if we denote by $g$ the degree of $a_{f}(p)$ and by $a_{f, 1}(p), \ldots, a_{f, g}(p)$ all of the conjugates of $a_{f}(p)$ (including $a_{f}(p)$ itself), then,

$$
\prod_{i=1}^{g}\left|a_{f, i}(p)\right| \geq 1
$$

By Deligne's proof of the Ramanujan-Petersson conjecture for $f$ [Del71], $a_{f}(p)$ is the sum of two $p$-Weil numbers of weight $k-1$, so $\left|a_{f, i}(p)\right| \leq 2 p^{\frac{k-1}{2}}$ for all $i$. Therefore,

$$
\prod_{i=1}^{g}\left|a_{f, i}(p)\right| \leq\left|a_{f}(p)\right|\left(2 p^{\frac{k-1}{2}}\right)^{g-1}
$$

and the claim follows.

Remark 2. If $\Gamma_{f} \leq \operatorname{Gal}\left(K_{f} / \mathbb{Q}\right)$ is the group of inner twists of $f$ (see [Rib80, Section 3], [Rib85, Section 3]), then the proof of Proposition 1 shows that the lower bound can actually be improved by a factor of $\left|\Gamma_{f}\right|$ (or even $\left|\Gamma_{f}\right|^{2}$ if $\chi(p) \in \mathbb{Q}^{\times}$). In the case 
$k=2, \chi=1, N$ square-free, there are no nontrivial inner twists, but otherwise it is believed that $\left|\Gamma_{f}\right|$ could become large; if $\chi^{2} \neq 1$, there is always a nontrivial inner twist given by conjugation.

We will now use the equidistribution of Hecke eigenvalues to exhibit a newform $f \in S_{k}\left(\Gamma_{0}(N), \chi\right)^{\text {new }}$ for which $a_{f}(p)$ is abnormally small, yet non-zero. This will therefore give a lower bound for the degree of $\mathbb{Q}\left(a_{f}(p)\right)$ and thus a lower bound for the degree of $K_{f}$.

Lemma 1. Let $p \nmid N$. There exists a newform $f \in S_{k}\left(\Gamma_{0}(N), \chi\right)^{\text {new }}$ such that,

$$
0<\left|a_{f}^{\prime}(p)\right| \leq \frac{\pi}{2} \cdot \frac{p+1}{p} \cdot \frac{\log p}{\log N}
$$

for all sufficiently large $N$ (in terms of $k$ ).

Proof. Let $B_{k}\left(\Gamma_{0}(N), \chi\right)$ be the $\overline{\mathbb{Q}}$-basis of $S_{k}\left(\Gamma_{0}(N), \chi\right)^{\text {new }}$ composed of the $d_{k, N, \chi}$ newforms at level $N$. For $(n, N)=1$, let us also normalize Hecke operators acting on $S_{k}\left(\Gamma_{0}(N), \chi\right)^{\text {new }}$ as $T_{n}^{\prime}:=T_{n} /\left(2 n^{\frac{k-1}{2}} \sqrt{\chi(n)}\right)$. By [Ser97, Sections 5.1, 5.3], the normalized eigenvalues $\left(a_{f}^{\prime}(p)\right)_{f \in B_{k}\left(\Gamma_{0}(N), \chi\right)}$ are distributed in $[-1,1]$ as $N \rightarrow \infty$ according to a measure converging to the Sato-Tate measure as $p \rightarrow \infty$.

For $A \in(0,1)$, let us give a lower bound on

$$
C_{k, N, \chi}(A):=\frac{\left|\left\{f \in B_{k}\left(\Gamma_{0}(N), \chi\right): 0<\left|a_{f}^{\prime}(p)\right| \leq A\right\}\right|}{d_{k, N, \chi}} .
$$

If the nebentypus is trivial and we do not necessarily want to find a form that is new, we can directly apply [MS09, Theorem 19] to get (3) below. In general, [MS09, Theorem 8, Lemma 17, Section 10] show that for any $M \geq 1$,

$$
\begin{aligned}
& \left|C_{k, N, \chi}(A)-2 \int_{0}^{A} F(-x) d x\right| \\
& \leq \frac{1}{M+1}+\sum_{1 \leq|m| \leq M}\left(\frac{1}{M+1}+\min \left(2 A, \frac{1}{\pi|m|}\right)\right)\left|\frac{\operatorname{tr}\left(T_{p^{|m|}}^{\prime}-T_{p|m|-2}^{\prime}\right)}{d_{k, N, \chi}}-c_{m}\right|,
\end{aligned}
$$

where $c_{m}=\lim _{k+N \rightarrow \infty} \operatorname{tr}\left(T_{p|m|}^{\prime}-T_{p^{|m|-2}}^{\prime}\right) / d_{k, N, \chi}$ and $F(x)=\sum_{m \in \mathbb{Z}} c_{m} e(m x)$, with the convention that $T_{n}^{\prime}=0$ if $n<1$. The Eichler-Selberg trace formula for $S_{k}\left(\Gamma_{0}(N), \chi\right)$ [Ser97, (34)] and [Ser97, Section 5.3] gives that,

$$
\begin{aligned}
\operatorname{tr} T_{p^{m}}^{\prime}=\sum_{N_{1} \mid N} d^{*}\left(N / N_{1}\right)( & A_{\text {main }}\left(k, N_{1}, T_{p^{m}}^{\prime}\right)+A_{\text {ell }}\left(k, N_{1}, \chi, T_{p^{m}}^{\prime}\right) \\
& \left.\quad+A_{\text {hyp }}\left(k, N_{1}, \chi, T_{p^{m}}^{\prime}\right)+\underset{\substack{k=2 \\
\chi=1}}{A_{\mathrm{par}}}\left(k, N_{1}, T_{p^{m}}^{\prime}\right)\right),
\end{aligned}
$$


for any $m \geq 1$, with the main, elliptic, hyperbolic and parabolic terms given in [Ser97, $(35,39,45,47)]$, and where $d^{*}$ is the multiplicative function defined by $d^{*}(\ell)=-2$, $d^{*}\left(\ell^{2}\right)=1$, and $d^{*}\left(\ell^{\alpha}\right)=0$ for $\ell$ a prime and $\alpha \geq 3$ an integer. By [Ser97, (35)],

$$
\sum_{N_{1} \mid N} d^{*}\left(N / N_{1}\right) A_{\text {main }}\left(k, N_{1}, T_{p^{m}}^{\prime}\right)=\frac{\psi(N)^{\text {new }}(k-1)}{12} \cdot p^{-m / 2} \cdot \delta_{m \text { even }},
$$

where $\psi(N)^{\text {new }}=\sum_{N_{1} \mid N} d^{*}\left(N / N_{1}\right) N_{1} \prod_{\ell \mid N_{1}}(1+1 / \ell)$, and by [MS09, Section 9],

$$
F(x)=\frac{\psi(N)^{\text {new }}(k-1)}{12 d_{k, N, \chi}} \cdot \frac{2(p+1)}{\pi} \cdot \frac{\sqrt{1-x^{2}}}{p+2+1 / p-4 x^{2}} .
$$

By [Ser97, $(44,46,48)]$, we find as in $[\operatorname{MS} 09,(8)]$ that for any $N_{1} \mid N$,

$$
\begin{aligned}
\left|A_{\mathrm{ell}}\left(k, N_{1}, \chi, T_{p^{m}}^{\prime}\right)\right| & \leq \frac{4 e}{\log 2} \cdot 2^{\omega\left(N_{1}\right)} p^{3 m / 2} \log \left(4 p^{m / 2}\right), \\
\left|A_{\mathrm{hyp}}\left(k, N_{1}, \chi, T_{p^{m}}^{\prime}\right)-A_{\mathrm{hyp}}\left(k, N_{1}, \chi, T_{p^{m-2}}^{\prime}\right)\right| & \leq \sqrt{N_{1}} \tau\left(N_{1}\right), \\
\left|A_{\mathrm{par}}\left(k, N_{1}, T_{p^{m}}^{\prime}\right)-A_{\mathrm{par}}\left(k, N_{1}, T_{p^{m-2}}^{\prime}\right)\right| & \leq p^{m / 2} .
\end{aligned}
$$

Moreover, we note that $\left|d^{*}(n)\right| \leq 2^{\omega(n)} \leq \tau(n) \ll_{\varepsilon} n^{\varepsilon}$ for all integers $n$ (see [Ser97, (52)]). Hence, this yields with (2)

$$
\begin{aligned}
C_{k, N, \chi}(A) \geq & \frac{\psi(N)^{\text {new }}(k-1)}{12 d_{k, N, \chi}} \cdot \frac{4(p+1)}{\pi} \int_{0}^{A} \frac{\sqrt{1-x^{2}}}{p+2+1 / p-4 x^{2}} d x-\frac{1}{M+1} \\
& -c(\varepsilon) N^{\varepsilon}\left(\frac{4 e}{\log 2} \cdot \frac{p^{3 M / 2}}{d_{k, N, \chi}} \cdot \log \left(4 p^{M / 2}\right)-\frac{\sqrt{N}}{d_{k, N, \chi}}-\delta_{\substack{k=2 \\
\chi=1}} \cdot \frac{p^{M / 2}}{d_{k, N, \chi}}\right),
\end{aligned}
$$

for any $\varepsilon>0$, with $c(\varepsilon)>0$ a constant depending only on $\varepsilon$. As in [Ser97, $(61,62)$,

$$
d_{k, N, \chi}=\frac{k-1}{12} \cdot \psi(N)^{\text {new }}+O\left(N^{1 / 2+\varepsilon}\right),
$$

therefore given $\varepsilon<1 / 100$ positive, as long as $M \leq(2 / 3-3 \varepsilon) \log (N) / \log p$, all the three terms in (4) are less than $c^{\prime}(\varepsilon) N^{-\varepsilon / 100}$ for all $N$ and some constants $c^{\prime}(\varepsilon)$ depending only on $\varepsilon^{1}$.

By a Taylor expansion at $x=0$,

$$
\int_{0}^{A} \frac{\sqrt{1-x^{2}}}{p+2+1 / p-4 x^{2}} d x=\frac{p}{(p+1)^{2}} \cdot A \cdot(1+O(A)),
$$

\footnotetext{
${ }^{1}$ The choice of $M$ is motivated by the fact that the growth of (4) is dominated by the first term in (4) which is roughly of size $N^{\varepsilon} p^{3 M / 2} N^{-1}$. Thus it is sufficient to choose $M$ so that this term is negligible, that is $p^{3 M / 2} N^{\varepsilon-1} \ll N^{-\varepsilon}$.
} 
therefore

$$
C_{k, N, \chi}(A) \geq \frac{4}{\pi} \cdot \frac{p}{p+1} \cdot A(1+O(A))-\frac{1}{M+1}-\frac{c^{\prime}(\varepsilon)}{N^{\varepsilon / 100}} .
$$

Hence, given $\varepsilon>0$, choosing $A$ so that,

$$
\frac{4}{\pi} \cdot \frac{p}{p+1} \cdot A>\left(\frac{3}{2}+\varepsilon\right) \cdot \frac{\log p}{\log N}>\frac{1+\varepsilon}{M+1}
$$

ensures that $C_{k, N, \chi}(A)>0$ for all sufficiently large $N$. In particular fixing a sufficiently small $\varepsilon>0$ we see that for all $N$ large enough any

$$
A>\frac{\pi}{2} \cdot \frac{p+1}{p} \cdot \frac{\log p}{\log N}
$$

is acceptable.

Theorem 1 and Theorem 2 now follows from combining Proposition 1 and Lemma 1 and specializing accordingly.

\section{Proof of Theorem 3}

For $k \geq 2$ and $N \geq 1$ square-free, let $f \in S_{k}\left(\Gamma_{0}(N), \chi\right)$ be a newform. We factor the character $\chi$ as $\prod_{p \mid N} \chi_{p}$ with $\chi_{p}:(\mathbb{Z} / p)^{\times} \rightarrow \mathbb{C}^{\times}$a character modulo $p$. The idea behind Theorem 3 is inspired by [CK06], where Choie and Kohnen show that the non-diagonalizability of a "bad" Hecke operator $T_{p}$ (i.e. with $p \mid N$ ) implies that $\sqrt{p} \in \mathbb{Q}\left(a_{n}(f): n \geq 1\right)$, and hence that this field has degree at least $2^{s}$ if $s$ such operators are non-diagonalizable.

Let

$$
N_{2}=\prod_{\substack{p \mid N \\ \chi_{p}=1}} p
$$

and write $N=N_{1} N_{2}$, with $\left(N_{1}, N_{2}\right)=1$ since $N$ is square-free. It follows that $\chi=\chi_{N_{1}} \chi_{N_{2}}$ with $\chi_{N_{1}}$ a primitive character of modulus $N_{1}$ and $\chi_{N_{2}}=1$ the principal character modulo $N_{2}$. Our argument is based on the Atkin-Lehner operators

$$
W_{p}: S_{k}\left(\Gamma_{0}(N), \chi\right) \rightarrow S_{k}\left(\Gamma_{0}(N), \bar{\chi}_{p} \chi_{N / p}\right), p \mid N
$$

where $\chi_{N / p}=\prod_{\ell \mid N / p} \chi_{\ell}$ and on the properties of the pseudo-eigenvalues $\lambda_{p}(f)$ studied by Atkin and Li [Li74, AL78]. Examining these elements gives bounds on the degrees of Fourier coefficients $a_{f}(p)$ at "bad" primes $p \mid N_{2}$. In turn, this yields lower bounds on $\operatorname{deg} K_{f}$ since:

Lemma 2. We have $K_{f}=\mathbb{Q}\left(a_{f}(n): n \geq 1\right)$. 
Proof. Let $K:=\mathbb{Q}\left(a_{f}(n): n \geq 1\right)$ and let $L$ be its Galois closure. By the Hecke relations $a_{f}(p)^{2}=a_{f}\left(p^{2}\right)-p^{k-1} \chi(p)$ for all $p \nmid N$, we have the tower of extensions $\mathbb{Q}\left(\zeta_{\text {ord } \chi}\right) \subset K_{f} \subset K \subset L$. By Galois theory, it suffices to show that $\operatorname{Gal}\left(L / K_{f}\right) \subset$ $\operatorname{Gal}(L / K)$. To that effect, let $\sigma \in \operatorname{Gal}\left(L / K_{f}\right)$. By the fact that $\chi^{\sigma}=\chi$ and [DI95, Corollary 12.4.5], $f^{\sigma}$ is a newform in $S_{k}\left(\Gamma_{0}(N), \chi\right)$ whose Fourier coefficients coincide with those of $f$ at all integers co-prime to $N$. By strong multiplicity one [DI95, Theorem 6.2.3], $f=f^{\sigma}$, so that $\sigma$ fixes all coefficients of $f$, i.e. $\sigma$ fixes $K$.

Recall that for $p \mid N$, the pseudo-eigenvalue $\lambda_{p}(f) \in \mathbb{C}$ is defined by the equation

$$
W_{p} f=\lambda_{p}(f) g,
$$

where $g \in S_{k}\left(\Gamma_{0}(N), \bar{\chi}_{p} \chi_{N / p}\right)$ is a newform (see [AL78, p.224]) given by

$$
a_{g}(\ell)= \begin{cases}\bar{\chi}_{p}(\ell) a_{f}(\ell) & : \ell \neq p \\ \chi_{N / p}(p) \overline{a_{f}(p)} & : \ell=p\end{cases}
$$

for primes $\ell([\mathrm{AL} 78,(1.1)])$.

In general, we only know that the pseudo-eigenvalue $\lambda_{p}(f)$ is algebraic with modulus 1 ([AL78, Theorem 1.1]). However, under additional assumptions on $\chi$, we have the following information on its field of definition:

Lemma 3. Let $p \mid N_{2}$. Then, $\lambda_{p}(f) \in \mathbb{Q}\left(\zeta_{2} \operatorname{ord}(\chi)\right)$.

Proof. From the identity $W_{p}^{2}=\chi_{p}(-1) \bar{\chi}_{N / p}(p)$ id ([AL78, Proposition 1.1]), we get that

$$
\lambda_{p}(f) \lambda_{p}(g)=\chi_{p}(-1) \bar{\chi}_{N / p}(p)= \pm \bar{\chi}_{N / p}(p) .
$$

Since $p \mid N_{2}$ we have $\chi_{p}=1$, so that $g \in S_{k}\left(\Gamma_{0}(N), \chi\right)$, and $a_{g}(\ell)=a_{f}(\ell)$ for all prime $\ell \neq p$, by (5). By strong multiplicity one, we get $g=f$. By (6), we obtain $\lambda_{p}(f)^{2}=\bar{\chi}_{N / p}(p)$ and thus the claim.

The next ingredient is the explicit determination of $\lambda_{f}(p)$ in terms of $a_{f}(p)$ by Atkin and Li.

Lemma 4. Let $p \mid N_{2}$. Then $a_{f}(p) \neq 0$ and

$$
\lambda_{p}(f)=-\frac{p^{k / 2-1}}{a_{f}(p)} .
$$

Proof. The fact that $a_{f}(p) \neq 0$ is [Li74, Theorem 3(ii)], and the formula for the eigenvalue is [AL78, Theorem 2.1].

Proof of Theorem 3. By Lemmas 2, 3 and 4, we get

$$
\left\{p^{k / 2}: p \mid N_{2}\right\} \quad \subset \quad K_{f}\left(\zeta_{2 \operatorname{ord}(\chi)}\right)
$$


Since $L:=\mathbb{Q}\left(\zeta_{\text {ord }(\chi)}\right) \subset K_{f}$, we have

$$
\begin{aligned}
{\left[K_{f}: \mathbb{Q}\right] } & \geq \frac{1}{2} \cdot\left[K_{f}\left(\zeta_{2 \operatorname{ord}(\chi)}\right): \mathbb{Q}\right] \\
& =\frac{1}{2} \cdot\left[K_{f}\left(\zeta_{2 \operatorname{ord}(\chi)}\right): L\right] \cdot \varphi(\operatorname{ord}(\chi)),
\end{aligned}
$$

where the last factor is the trivial bound.

The square roots of odd primes $p \mid \operatorname{ord}(\chi)$ belong to $L$. On the other hand, for $S:=\left\{\sqrt{p}: p \mid N_{2}, p \nmid 2 \operatorname{ord}(\chi)\right\} \subset K_{f}\left(\zeta_{2} \operatorname{ord}(\chi)\right)$, we have

$$
\left[K_{f}\left(\zeta_{2 \operatorname{ord}(\chi)}\right): L\right] \geq[L(S): L]=2^{|S|}
$$

by [Hil98, Theorem 87], and the claim follows.

Remark 3. Since the character $\chi_{p}$ is primitive for $p \mid N_{1}$, [Li74, Theorem 3(ii)] and [AL78, Theorem 2.1, Proposition 1.4] show that $\lambda_{p}(f)=p^{k / 2-1} g\left(\chi_{p}\right) / a_{f}(p)$, with $g\left(\chi_{p}\right)$ the Gauss sum attached to $\chi_{p}$. The degree of $p^{k / 2-1} g\left(\chi_{p}\right)$ over $\mathbb{Q}$ can be determined precisely, however we have no information about the field of definition of $\lambda_{p}(f)$, except the fact that it is a root of unity. If we could show that it belongs to a small extension of $K_{f}$, in the same way as we did for $\lambda_{p}(f)$ with $p \mid N_{2}$, then we could add a factor as large as ord $(\chi)$ to the lower bound of Theorem 3 , including when $k$ is even.

\section{REFERENCES}

[AL78] A.O.L. Atkin and W. W. Li. Twists of newforms and pseudo-eigenvalues of $W$-operators. Inventiones Mathematicae, 1978.

[BM16] N. Billerey and R. Menares. On the modularity of reducible $\bmod l$ Galois representations. Math. Res. Lett., 23(1):15-41, 2016.

[Bru95] A. Brumer. The rank of $J_{0}(N)$. Astérisque, (228):3, 41-68, 1995. Columbia University Number Theory Seminar (New York, 1992).

[CE04] F. Calegari and M. Emerton. The Hecke algebra $T_{k}$ has large index. Math. Res. Lett., 11(1):125-137, 2004.

[CF99] J. B. Conrey and D. W. Farmer. Hecke operators and the nonvanishing of $L$-functions. In Topics in number theory (University Park, PA, 1997), volume 467 of Math. Appl., pages 143-150. Kluwer Acad. Publ., Dordrecht, 1999.

[CK06] Y. Choie and W. Kohnen. Diagonalizing "bad" Hecke operators on spaces of cusp forms. In W. Zhang and Y. Tanigawa, editors, Number theory: tradition and modernization, pages 23-26. Springer US, Boston, MA, 2006.

[Del71] P. Deligne. Formes modulaires et représentations l-adiques. In Séminaire Bourbaki. Vol. 1968/69: Exposés 347-363, volume 175 of Lecture Notes in Math., pages Exp. No. 355, 139-172. Springer, Berlin, 1971.

[DI95] F. Diamond and J. Im. Modular forms and modular curves. In Seminar on Fermat's Last Theorem (Toronto, ON, 1993-1994), volume 17 of CMS Conf. Proc., pages 39-133. Amer. Math. Soc., Providence, RI, 1995. 
[DJUR15] L. V. Dieulefait, J. Jiménez Urroz, and K. A. Ribet. Modular forms with large coefficient fields via congruences. Res. Number Theory, 1:Art. 2, 14, 2015.

[DT16] L. Dieulefait and P. Tsaknias. Possible connection between a generalized Maeda's conjecture and local types. preprint arXiv:1608.05285, 2016.

[Hil98] D. Hilbert. The theory of algebraic number fields. Springer Berlin Heidelberg, 1998.

[HM97] H. Hida and Y. Maeda. Non-abelian base change for totally real fields. Pacific J. Math., (Special Issue):189-217, 1997. Olga Taussky-Todd: in memoriam.

[Li74] W. W. Li. Newforms and Functional Equations. Mathematische Annalen, 212, 1974.

[LS18] M. Lipnowski and G. J. Schaeffer. Detecting large simple rational Hecke modules for $\Gamma_{0}(N)$ via congruences. International Mathematics Research Notices, page rny190, 2018.

[Mat10] MathOverflow. Galois orbits of newforms with prime power level, May 2010. https://mathoverflow. net/questions/24923.

[MS09] M. R. Murty and K. Sinha. Effective equidistribution of eigenvalues of Hecke operators. Journal of Number Theory, 129(3):681-714, 2009.

[Rib80] K. A. Ribet. Twists of modular forms and endomorphisms of abelian varieties. Mathematische Annalen, 253(1):43-62, 1980.

[Rib85] K. A. Ribet. On $\ell$-adic representations attached to modular forms. II. Glasgow Mathematical Journal, 27:185-194, 1985.

[Roy00] E. Royer. Facteurs Q-simples de $J_{0}(N)$ de grande dimension et de grand rang. Bull. Soc. Math. France, 128(2):219-248, 2000.

[Ser97] J-P. Serre. Rpartition asymptotique des valeurs propres de l'oprateur de Hecke $T_{p}$. Journal of the American Mathematical Society, 10(1):75-102, 1997.

[Tsa14] P. Tsaknias. A possible generalization of Maedas conjecture. In Gebhard Bckle and Gabor Wiese, editors, Computations with modular forms, volume 6, pages 317-329. Springer International Publishing, Cham, 2014.

Dipartimento di Matematica, Università di Genova, via Dodecaneso 35, 16146 GenOVA, ITALY

E-mail address: bettin@dima.unige.it

Centre de Recherches mathématiques, Université de Montréal, Montréal, Canada

E-mail address: corentin.perretgentil@gmail.com

Caltech, Department of Mathematics, 1200 E California Blvd, Pasadena, CA, 91125, USA

E-mail address: maksym.radziwill@gmail.com 